Tôhoku Math. Journ.

$32(1980)$, 447-452.

\title{
THE EXPONENT OF CONVERGENCE OF POINCARÉ SERIES ON SOME KLEINIAN GROUPS
}

\author{
TOHru AKaza and Harushi Furusawa \\ (Received October 9, 1979, revised January 14, 1980)
}

1. Let us denote by $G$ and $\Lambda(G)$ a finitely generated Kleinian group and its limit set, respectively. Let

$$
S_{i}(z)=\frac{a_{i} z+b_{i}}{c_{i} z+d_{i}}, \quad\left(a_{i} d_{i}-b_{i} c_{i}=1\right) \quad(i=0,1,2, \cdots)
$$

be any element of $G$, where $S_{0}(z)=z$ is the identity transformation. It is well known that the Poincaré series $P_{\mu}(z)=\sum_{S_{i} \in G}\left|c_{i} z+d_{i}\right|^{-\mu}(\mu>0)$ converges uniformly in any compact domain contained in the region of discontinuity if and only if $\sum_{i=1}^{\infty}\left|c\left(S_{i}\right)\right|^{-\mu}<+\infty$, where $c\left(S_{i}\right)=c_{i}$. The quantity

$$
\begin{aligned}
P(G) & =\inf \left\{\mu>0 ; \sum_{i=1}^{\infty}\left|c_{i} z+d_{i}\right|^{-\mu}<+\infty, z \notin \Lambda(G)\right\} \\
& =\inf \left\{\mu>0 ; \sum_{i=1}^{\infty}\left|c\left(S_{i}\right)\right|^{-\mu}<+\infty, S_{i}(\infty) \neq \infty\right\},
\end{aligned}
$$

is independent of $z(\notin \Lambda(G))$ and we shall call $P(G)$ the exponent of convergence for $G$. The Hausdorff dimension of the limit set $\Lambda(G)$ of $G$ is also defined as the non-negative number

$$
d(\Lambda(G))=\inf \left\{\eta ; M_{\eta}(\Lambda(G))=0\right\},
$$

where $M_{\eta}(\Lambda(G))$ denotes the $\eta$-dimensional measure of $\Lambda(G)$.

If $G$ is a finitely generated Fuchsian group of the first kind or of the second kind without parabolic elements or is a Schottky group, the following relation holds:

$$
d(\Lambda(G))=P(G) / 2, \quad([1],[7],[8]) .
$$

If $G$ is a finitely generated Fuchsian group of the second kind with parabolic elements, or more generally, if $G$ is a finitely generated Kleinian group and has a convex finite-sided fundamental polyhedron, then the following inequality holds:

$$
d(\Lambda(G)) \leqq P(G) / 2, \quad([3],[4],[7]) .
$$

2. Next we shall consider a finitely generated and non-elementary 
Kleinian group $G$, where $\infty$ is an ordinary point of $G$ fixed only by the identity $e \in G$. Let $F$ be the Ford fundamental domain of $G$. Denote by $T_{\lambda, r}(z)$ a hyperbolic or loxodromic transformation which satisfies the following conditions:

(i) $\left\{\bigcap_{g \in G-\{e\}} \operatorname{ext} I(g)\right\} \supset\left\{I\left(T_{\lambda, r}\right) \cup I\left(T_{\lambda, r}^{-1}\right)\right\}$,

(ii) the closure of the interior of $I\left(T_{\lambda, r}\right)$ has no common point with $I\left(T_{\lambda, r}^{-1}\right)$, where $I(S)$ denotes the isometric circle of $S, \lambda$ is the minimal distance from the boundary $\partial F$ to the centers of $I\left(T_{\lambda, r}\right)$ and $I\left(T_{\lambda, r}^{-1}\right)$ and $r$ is the radius of $I\left(T_{\lambda, r}\right)$.

As is well known, $r=\left|c\left(T_{\lambda, r}\right)\right|^{-1}=\left|c\left(T_{\lambda, r}^{-1}\right)\right|^{-1}$.

We consider the free product of $G$ and a cyclic group $\left\langle T_{\lambda, r}\right\rangle$ generated by $T_{\lambda, r}$ and write this in the form

$$
G_{\lambda, r}=G *\left\langle T_{\lambda, r}\right\rangle,
$$

which is also a finitely generated Kleinian group.

Next we shall consider a subgroup $G_{\lambda, r}^{\prime}$ of $G_{\lambda, r}$ generated by all elements of the form $p T_{\lambda, r}^{\varepsilon} p^{-1}$, with $p \in G, \varepsilon= \pm 1$. We can easily verify that $G_{\lambda, r}^{\prime}$ is a free subgroup of $G_{\lambda, r}$. For any element $g \in G_{\lambda, r}$ there exist $p, g^{\prime}$ and $g^{\prime \prime}$ satisfying $g=p g^{\prime}=g^{\prime \prime} p$, where $p \in G, g^{\prime}, g^{\prime \prime} \in G_{\lambda, r}$. Since $g G_{\lambda, r}^{\prime} g^{-1} \subset G_{\lambda, r}^{\prime}$ for each $g \in G_{\lambda, r}$, it is easily seen that $G_{\lambda, r}^{\prime}$ is a normal subgroup of $G_{\lambda, r}$.

Then we have the following theorem.

THEOREM 1. Let $G_{\lambda, r}$ and $G_{\lambda, r}^{\prime}$ be the groups defined above. Then

$$
\Lambda\left(G_{\lambda, r}\right)=\Lambda\left(G_{\lambda, r}^{\prime}\right) .
$$

The proof of this theorem is similar to the one pin the case of the Fuchsian groups (see Greenberg [6]).

3. Now let us discuss the relation among the three exponents $P(G)$, $P\left(G_{\lambda, r}\right)$ and $P\left(G_{\lambda, r}^{\prime}\right)$ defined by (1). Since $G_{\lambda, r}^{\prime}$ and $G$ are subgroups of $G_{\lambda, r}$, it is obvious that $P\left(G_{\lambda, r}^{\prime}\right) \leqq P\left(G_{\lambda, r}\right)$ and $P(G) \leqq P\left(G_{\lambda, r}\right)$. But the following problem is still open: Which is larger, $P\left(G_{\lambda, r}^{\prime}\right)$ or $P(G)$ ? In this paper we shall investigate the behavior of $P\left(G_{\lambda, r}\right)$, when $\lambda$ (or $r$ ) tends to infinity (or 0).

Take any number $\mu(>P(G))$. Denote by $Q_{G}(\mu)$ the series $\sum_{S_{i} \in G-(e)}\left|c\left(S_{i}\right)\right|^{-\mu}$. Then two cases may occur:

(i ) $\lim _{\mu \rightarrow P(G)} Q_{G}(\mu)=+\infty$,

(ii) $\lim _{\mu \rightarrow P(G)} Q_{G}(\mu)<+\infty$.

It is conjectured that only the case (i) holds for any Kleinian group, because we have the examples for (i) (hyperbolic and parabolic cyclic 
groups, Fuchsian groups of the first kind and the Schottky groups) and on the other hand we have no example for (ii) except for the finite groups (elliptic cyclic groups) ([2], [3], [5]).

If $G$ is a finite group, for example, an elliptic cyclic group, it is obvious that $\lim _{\mu \rightarrow P(G)} Q_{G}(\mu)<+\infty$, and in this case $G_{\lambda, r}^{\prime}$ is a Schottky group. We can easily verify that $0=P(G)<P\left(G_{\lambda, r}^{\prime}\right)$.

4. Statement of Theorems. We shall prove the following theorems about the exponents of convergence.

THEOREM 2. Suppose that $Q_{G}\left(\mu_{1}\right)<+\infty$ and take and fix any number $r_{1}<1$ (or $\left.\lambda_{1}>1\right)$. Then there exists $\lambda^{*}$ (or $\left.r^{*}\right)$ depending on $\mu_{1}$ and $r_{1}\left(\right.$ or $\left.\lambda_{1}\right)$ such that for any $\lambda\left(\geqq \lambda^{*}\right)$ (or $\left.r\left(\leqq r^{*}\right)\right)$

$$
P\left(G_{\lambda, r_{1}}\right) \leqq \mu_{1} \text {. }
$$

THEOREM 3. If $\lambda$ tends to $+\infty$ with a fixed $r(<1)$ (or if $r$ tends to zero with a fixed $\lambda(>1)$ ), then $P\left(G_{\lambda, r}\right)$ tends to $P(G)$.

THEOREM 4. Let $G$ be a Schottky group or a finitely generated Fuchsian group of the first kind or of the second kind without parabolic elements. If $\lambda$ tends to $+\infty$ with a fixed $r<1$ (or if $r$ tends to zero with a fixed $\lambda>1)$, then $d\left(\Lambda\left(G_{\lambda, r}\right)\right)$ tends to $d(\Lambda(G))$.

5. Preliminaries for the proof of the theorems. For brevity, we put $T=T_{\lambda, r}, G^{*}=G_{\lambda, r}$ and $G^{\prime}=G_{\lambda, r}^{\prime}$. Any element $g$ of $G^{*}-G$ is expressed in the form

$$
g=q_{n} T^{\varepsilon_{n}} q_{n-1} T^{\varepsilon_{n-1}} \cdots q_{1} T^{\varepsilon_{1}} q_{0} \quad\left(q_{j} \in G, \varepsilon_{j}= \pm 1\right),
$$

where $q_{j} \neq e$ for an integer $j(1 \leqq j \leqq n-1)$ with $T^{\varepsilon_{j+1}} T^{\varepsilon_{j}}=e$. Here we call the number $n$ the grade of $g$ in $G^{*}$ and we often write $g=g(n)$ $(n \geqq 1)$. Each element of $G$ is considered as an element of grade 0 and is denoted by $g(0)$. It is easily seen by (4) that for any element $g \in G^{*}-G$ there exist a unique $g^{\prime} \in G^{\prime}$ and a unique $p \in G$ satisfying $g=p g^{\prime}=\left(q_{n} q_{n-1} \cdots q_{0}\right) h(n)$, where $h(n)$ denotes an element of grade $n$ in $G^{\prime}$.

Consider the subset

$$
E(n)=\left\{p h(n) ; p \in G, h(n) \in G^{\prime}\right\}
$$

of $G^{*}$. For brevity we express $g$ in (4) by

$$
g=q_{n} g^{\prime}(n) q_{0},
$$

where $g^{\prime}(n)=T^{\varepsilon_{n}} q_{n-1} T^{\varepsilon_{n-1}} \cdots q_{1} T^{\varepsilon_{1}}$. Denote by $\omega\left(g^{\prime}(n)\right)$ the cardinality of the set $\left\{j ; q_{j}=e, 1 \leqq j \leqq n-1\right\}$ for $g^{\prime}(n)$ in (5). Then, if $\mu>P(G)$, 
we have

$$
\begin{aligned}
\boldsymbol{Q}_{G^{*}}(\mu) & =\sum_{g \in G^{*}-\{e\}}|c(g)|^{-\mu}=\sum_{n=0}^{\infty} \sum_{g \in E(n)}|c(g)|^{-\mu} \\
& =\sum_{g(0) \in G-\{e\}}|c(g(0))|^{-\mu}+\sum_{n=1}^{\infty}\left\{\sum_{k=0}^{n-1} \sum_{\omega\left(g^{\prime}(n)\right)=k}\left|c\left(q_{n} g^{\prime}(n) q_{0}\right)\right|^{-\mu}\right\} .
\end{aligned}
$$

It is well known that

$$
\left|c\left(S_{1} S_{2}\right)\right|^{-1}=\left|c\left(S_{1}\right)\right|^{-1}\left|c\left(S_{2}\right)\right|^{-1}\left|S_{1}^{-1}(\infty)-S_{2}(\infty)\right|^{-1}
$$

for two linear transformations $S_{1}$ and $S_{2}$ with $c\left(S_{1}\right) \neq 0, c\left(S_{2}\right) \neq 0$ (see [5]). For the simplicity of the calculation we assume that

$$
\inf _{m n<0}\left|T^{m}(\infty)-T^{n}(\infty)\right| \geqq 1 \text {. }
$$

Now we shall estimate the term $\sum_{\omega\left(g^{\prime}(n)=k\right.}\left|c\left(q_{n} g^{\prime}(n) q_{0}\right)\right|^{-\mu}$. By taking a number $\lambda$ sufficiently large, we may assume $\lambda \geqq 1$. If $q_{n} \neq e$ and $q_{0} \neq e$, then, using (6), we have for $n \geqq 1$

$$
\left|c\left(q_{n} g^{\prime}(n) q_{0}\right)\right|^{-1} \leqq \lambda^{-2} \times\left|c\left(q_{0}\right)\right|^{-1} \times\left|c\left(q_{n}\right)\right|^{-1} \times\left|c\left(g^{\prime}(n)\right)\right|^{-1},
$$

because $g^{\prime}(n)(\infty)$ lies inside $I(T)$ or $I\left(T^{-1}\right)$. When just one of $q_{0}$ and $q_{n}$ is the identity, say $q_{n}=e$, we have

$$
\left|c\left(g^{\prime}(n) q_{0}\right)\right|^{-1} \leqq \lambda^{-1}\left|c\left(q_{0}\right)\right|^{-1}\left|c\left(g^{\prime}(n)\right)\right|^{-1} .
$$

Next we estimate $\left|c\left(g^{\prime}(n)\right)\right|^{-1}$. If $\omega\left(g^{\prime}(n)\right)=k(0 \leqq k \leqq n-1)$, it is easily seen from (6) and (7) that

$$
\left|c\left(g^{\prime}(n)\right)\right|^{-1} \leqq\left(\Pi^{\prime}\left|c\left(q_{j}\right)\right|^{-1}\right)|c(T)|^{-n} \lambda^{-2(n-k-1)},
$$

where $\Pi^{\prime}$ denotes the product of $(n-k-1)$ terms taken for $j$ with $q_{j} \neq e(1 \leqq j \leqq n-1)$. Since $\mu>P(G)$, we have from (10)

$$
\begin{aligned}
\sum_{\omega\left(g^{\prime}(n)\right)=k}\left|c\left(g^{\prime}(n)\right)\right|^{-\mu} \leqq{ }_{n-1} C_{k} \times 2^{n-k} & \left(\sum_{q \in G-\{e\}}|c(q)|^{-\mu}\right)^{n-k-1} \\
& \times\left(|c(T)|^{-\mu}\right)^{n} \times\left(\lambda^{-\mu}\right)^{2(n-k-1)} .
\end{aligned}
$$

Combining (8) and (9) with (11), we have

$$
\left\{\begin{aligned}
A_{k} & =\sum_{\omega\left(g^{\prime}(n)\right)=k}\left|c\left(q_{n} g^{\prime}(n) q_{0}\right)\right|^{-\mu} \\
& \leqq{ }_{n-1} C_{k} \times 2^{n-k}\left(Q_{G}(\mu)\right)^{n-k+1} \times\left(|c(T)|^{-\mu}\right)^{n} \times\left(\lambda^{-\mu}\right)^{2(n-k)}, \quad\left(q_{n} \neq e, q_{0} \neq e\right), \\
B_{k} & =\sum_{\omega\left(g^{\prime}(n)\right)=k}\left|c\left(g^{\prime}(n) q_{0}\right)\right|^{-\mu} \\
& \leqq{ }_{n-1} C_{k} \times 2^{n-k}\left(Q_{G}(\mu)\right)^{n-k} \times\left(|c(T)|^{-\mu}\right)^{n} \times\left(\lambda^{-\mu}\right)^{2(n-k)-1}, \quad\left(q_{n}=e, q_{0} \neq e\right) .
\end{aligned}\right.
$$

From this we have

$$
\sum_{k=0}^{n-1} A_{k} \leqq 2 Q_{G}(\mu)^{2} \times\left(\lambda^{-2 \mu}\right)\left\{\sum_{k=0}^{n-1}{ }_{n-1} C_{k} \times\left(2 Q_{G}(\mu) \lambda^{-2 \mu}\right)^{n-k-1}\right\} \times\left(|c(T)|^{-\mu}\right)^{n}
$$




$$
=2 Q_{G}(\mu)^{2}\left(\lambda^{2 \mu}+2 Q_{G}(\mu)\right)^{-1}\left(1+2 Q_{G}(\mu) \lambda^{-2 \mu}\right)^{n}|c(T)|^{-\mu n}
$$

and

$$
\begin{aligned}
\sum_{k=0}^{n-1} B_{k} & \leqq 2 Q_{G}(\mu) \times\left(\lambda^{-\mu}\right)\left\{\sum_{k=0}^{n-1}{ }_{n-1} C_{k} \times\left(2 Q_{G}(\mu) \lambda^{-2 \mu}\right)^{n-k-1}\right\} \times\left(|c(T)|^{-\mu}\right)^{n} \\
& =2 Q_{G}(\mu) \lambda^{\mu}\left(\lambda^{2 \mu}+2 Q_{G}(\mu)\right)^{-1}\left(1+2 Q_{G}(\mu) \lambda^{-2 \mu}\right)^{n}|c(T)|^{-\mu n}
\end{aligned}
$$

Therefore by (11), (12) and (13) we can estimate $Q_{G^{*}}(\mu)$ as follows:

$$
\begin{aligned}
& Q_{G^{*}}(\mu) \leqq \sum_{g(0) \in G-\{e\}}|c(g(0))|^{-\mu}+\sum_{n=1}^{\infty}\left\{\sum_{k=0}^{n-1}\left(A_{k}+2 B_{k}+\sum_{\omega\left(g^{\prime}(n)\right)=k}\left|c\left(g^{\prime}(n)\right)\right|^{-\mu}\right)\right\} \\
& \leqq Q_{G}(\mu)+2\left(\lambda^{2 \mu}+2 Q_{G}(\mu)\right)^{-1}\left(\lambda^{\mu}+Q_{G}(\mu)\right)^{2} \\
& \times\left[\sum_{n=1}^{\infty}\left\{\left(1+2 Q_{G}(\mu) \lambda^{-2 \mu}\right)|c(T)|^{-\mu}\right\}^{n}\right] .
\end{aligned}
$$

REMARK. In a manner similar to the above we can obtain the following estimate of $Q_{G^{\prime}}(\mu)$ :

$$
\begin{aligned}
& Q_{G^{\prime}}(\mu)=\sum_{g^{\prime} \in G^{\prime}-\{e\}}\left|c\left(g^{\prime}\right)\right|^{-\mu} \leqq K(\lambda, \mu) \sum_{n=1}^{\infty}\left\{\left(1+2 Q_{G}(\mu) \lambda^{-2 \mu}\right)|c(T)|^{-\mu}\right\}^{n}, \\
& K(\lambda, \mu)=2\left(\lambda^{2 \mu}+2 Q_{G}(\mu)\right) \lambda^{2 \mu} .
\end{aligned}
$$

In view of Theorem 1, (14) and (15) it is very likely that $P\left(G^{*}\right)=P\left(G^{\prime}\right)$.

6. Proofs of Theorems. Proof of Theorem 2. Take and fix any positive number $r_{1}<1$. If we take $\lambda^{*}$ in such a way that

$$
r_{1}^{\mu_{1}}\left\{1+2 Q_{G}\left(\mu_{1}\right)\left(\lambda^{*}\right)^{-2 \mu_{1}}\right\}<1,
$$

then (14) implies that $Q_{G^{*}}\left(\mu_{1}\right)$ converges for $G^{*}=G_{\lambda, r_{1}}\left(\lambda \geqq \lambda^{*}\right)$. Hence $P\left(G^{*}\right) \leqq \mu_{1}$. When $\lambda_{1}>1$ is given, we take $r^{*}(<1)$ in such a way that

$$
\left(r^{*}\right)^{\mu_{1}}\left(1+2 Q_{G}\left(\mu_{1}\right) \lambda_{1}^{-2 \mu_{1}}\right)<1
$$

and we have the required result.

Proof of Theorem 3. Take any number $\mu_{1}$ with $P(G)<\mu_{1}<$ $P(G)+\varepsilon$ for any small number $\varepsilon(>0)$. Fix $r_{1}<1$ (or $\lambda_{1}>1$ ) arbitrarily and take $\lambda$ (or $r$ ) such that $\lambda>\lambda^{*}$ (or $r<r^{*}$ ), where $\lambda^{*}$ (or $r^{*}$ ) is determined by (16) (or (17)). Theorem 2 shows $P(G) \leqq P\left(G^{*}\right) \leqq \mu_{1}<$ $P(G)+\varepsilon$, where $G^{*}=G_{\lambda_{, r_{1}}}$ (or $G_{\lambda_{1}, r}$ ). Therefore we obtain the conclusion of Theorem 3.

Proof of Theorem 4. If $G$ is a Schottky group, it is obvious that $G_{\lambda, r}$ is also a Schottky group. By (2) and Theorem 3 it is clear that $d\left(\Lambda\left(G_{\lambda, r}\right)\right.$ ) tends to $d(\Lambda(G))$ for $\lambda \rightarrow+\infty$ (or $r \rightarrow 0$ ) with a fixed $r_{1}<1$ (or $\left.\lambda_{1}>1\right)$.

If $G$ is a finitely generated Fuchsian group of the first kind or of 
the second kind without parabolic elements, then $G_{\lambda, r}$ constructed in Section 2 is also a finitely generated Kleinian group and has a finitesided fundamental polyhedron. Thus we obtain from (3) that $P\left(G_{\lambda, r}\right) / 2 \geqq$ $d\left(\Lambda\left(G_{\lambda, r}\right)\right)$. Hence (2) shows $0 \leqq d\left(\Lambda\left(G_{\lambda, r}\right)\right)-d(\Lambda(G)) \leqq\left(P\left(G_{\lambda, r}\right)-P(G)\right) / 2$. Theorem 3 gives the desired result.

\section{REFERENCES}

[1] T. AKazA, Local property of the singular sets of some Kleinian groups, Tôhoku Math. J. 25 (1973), 1-22.

[2] T. Akaza and T. Shimazaki, The Hausdorff dimension of the singular sets of combination groups, Tôhoku Math. J. 25 (1973), 61-68.

[3] A. F. Beardon, The exponent of convergence of Poincare series, Proc. London Math. Soc. 18 (1968), 461-483.

[4] A. F. BEARdon aNd B. Maskit, Limit points of Kleinian groups and finite sided fundamental polyhedra, Acta Math. 132 (1974), 1-12.

[5] L. R. Ford, Automorphic Functions, 2nd ed. Chelsea, New York, 1951.

[6] L. GreEnberg, Finiteness theorems for Fuchsian and Kleinian groups, in Discrete Groups and Automorphic Functions, Acad. Press. New York, 1977, 199-257.

[7] S. T. Patterson, The limit set of a Fuchsian group, Acta Math. 136 (1976), 241-273.

[8] H. Peterson, Uber der Bereich absoluter Konvergenz der Poincaréschen Reihen, Acta Math. 80 (1948), 23-49.

$\begin{array}{lll}\text { Department of Mathematics } & \text { and } & \text { Department of Mathematics } \\ \text { Kanazawa University } & & \text { Kanazawa Women's Junior College } \\ \text { Kanazawa, 920 JaPaN } & \text { Kanazawa, 920 JapaN }\end{array}$

